\title{
Genetic Algorithm-Based Approach for Minimising Losses in Substrate-Integrated Waveguide
}

\author{
Hidayah Mansor ${ }^{1}$, Razi Abdul-Rahman ${ }^{2}$ \\ ${ }^{1,2}$ School of Mechanical Engineering, Universiti Sains Malaysia, 14300 Nibong Tebal, Pulau Pinang, Malaysia
}

\begin{tabular}{l} 
Article Info \\
\hline Article historys: \\
Received Aug 21, 2019 \\
Revised Mar 6, 2020 \\
Accepted Mar 24, 2020 \\
\hline
\end{tabular}

\section{Keywords:}

Millimeter-wave

Air-filled substrate integrated waveguide

Substrate integrated waveguide

Multi-objective genetic

algorithm

Clamped cubic spline

Optimization procedures

\begin{abstract}
The transitions in an air-filled substrate-integrated waveguide (SIW) are studied here for millimetre-wave applications. A good design of an air-filled SIW (AFSIW) must allow for minimum losses in its interconnects between the air-filled and dielectric-filled regions of the SIW. This paper assesses the influence of the geometry of transition taper in an AFSIW on the return and insertion losses using full-wave analysis of a complete AFSIW structure. The data from the return and transmission losses provide a basis for the optimisation of the design of the transition tapers. The optimisation approach uses the multi-objective genetic algorithm (GA) with full-wave analysis to find an optimum profile of the transition. Defining the profile of the transition taper with a clamped cubic spline as a phenotype, the developed procedure shows that further losses are possible within the prescribed frequency bands. Furthermore, the length of the transition taper can be significantly reduced while maintaining an optimal quality of signal transmission in the transition. The simulation results show the efficacy of the proposed strategy where the optimal taper geometry is shown to provide a wider band of operating frequencies with lower return loss compared to a more established taper geometry.
\end{abstract}

Copyright $@ 2019$ Institute of Advanced Engineering and Science. All rights reserved.

\section{Corresponding Author:}

Nur Hidayah Mansor,

School of Mechanical Engineering, Universiti Sains Malaysia,

14300 Nibong Tebal, Pulau Pinang,

Malaysia.

Email: menurhidayah@usm.my

\section{INTRODUCTION}

Since its inception about 15 years ago, substrate-integrated waveguide (SIW) technology is gaining more attention as a solution to an ever-increasing need of millimetre-wave devices to meet the demand for ubiquitous wireless networking. Retaining the good properties of rectangular waveguides while enabling mature manufacturing processes such as the printed circuit board (PCB) technique, SIWs are commonly accepted to be a competitive technology. Hence, SIW devices enable fabrication of a complete circuit including planar circuitry, transitions, rectangular waveguides, active components, and antennas in planar form using common planar processing techniques [1-5].

Consequently, various components based on the SIW technology have been proposed and applied in the recent years for operation in the microwave and millimetre-wave range, including filters, couplers, oscillators, slot-array antennas, six-port circuits, and circulators. However, one of the major issues in the design of SIW components is related to the minimization of losses, especially when operating in the millimetre-wave frequency range [6]. The mechanisms of loss in the SIW structures include conductor losses due to the finite conductivity of metallic walls, radiation losses due to the presence of gaps in the SIW structures along the side walls, and dielectric losses due to the loss tangent of the dielectric substrate [7]. The dielectric loss is typically the most significant contribution to losses in the millimetre-wave frequency range [6]. 
The earlier efforts at designing low-loss SIW involve material removal between sidewalls, thus effectively creating a rectangular waveguide structure in place of the dielectric region. One of the earlier proposals addresses the issue of fabrication of the hollow structure and characterization of the improvement in the dielectric loss in terms of the attenuation constant. Although the actual fabrication of this modified SIW has not been reported, their numerical and theoretical analyses affirm the idea of a hollow SIW to significantly reduce dielectric losses [8-9]. It was only until later that other groups proposed new designs having an air region in SIW were some with actual fabrications and experimental results. These include the so-called hollow SIW, empty SIW, and air-filled SIW (AFSIW), which are seemingly being developed independently [10-12].

Although the groups share a common approach similar to that proposed in [9], the AFSIW design addresses an important aspect of having a wideband operation in SIW through transition and interconnects [12]. Improving the wideband aspect in transition and interconnects of devices having different dielectric constants is essential in complete front-end integration and low-cost system manufacturing [13]. For this purpose, the new design of AFSIW and detailed characterization of the air-dielectric transition is quite successful [12]. Their idea is essentially a further development of the approach in [13], which focuses on transitions between multiple conventional SIWs with mixed low and high dielectric constants. Nevertheless, the new design in [12] is quite sensitive in its performance in terms of the return and insertion losses with respect to the length of transition. It is noteworthy to further study of this aspect, which is the focus of this paper.

As a wave propagates between two media, part of the wave, in general, is reflected, while another part is transmitted into the second medium. The mechanism of losses along the transition in waveguides has long been studied in [16-19]. However, in the context of SIW, wave scattering is more complex. Note that the previous attempts at improving the performance of SIW and analysis of losses in inhomogeneous media strongly indicate that there exists at least a unique shape of transition at a specific length that minimizes either the return or transmission loss. However, a closed-form description of such optimal shape is rather impossible to determine. In this paper, an optimisation procedure for an AFSIW transition taper using a multi-objective genetic algorithm (MO-GA) is developed to estimate the optimal shape of the transition. Since phenotypes are the decision variables in the GA procedure, a convenient approach is to construct the taper geometry in terms of cubic splines, where variables at the knots provide variations in the shape of the splines. Consequently, the number of internal knots provides the number of phenotypes.

In section 2, an AFSIW structure and its properties are discussed. Then, the transition of dielectric SIW to AFSIW operating at the Ka-band frequencies, i.e., 26-40 GHz, and U-band frequencies, i.e., 40-60 GHz, is presented in section 3. To create a new design of the transition taper with better losses while maintaining the quality of the transition, an optimisation procedure is detailed in section 4. Finally, the results of the optimal designs of the transition taper are analysed in section 5.

\section{RESEARCH METHOD}

An early proposal of realising a hollow region between the SIW sidewalls is based on a conventional printed circuit board (PCB) process using three layers of the dielectric substrate, where the core is removed by a specific width and shape between the metallic vias and is sandwiched between a pair of copper laminates [6]. Similar fabrication is used in AFSIW fabrication in [12], where the cross-sectional area of the three-layer substrate is depicted in Figure 1a. A part of the hollow region that constitutes the middle substrate can be seen in Figure $1 \mathrm{~b}$. This design is used in the subsequent modeling and analysis in this study.

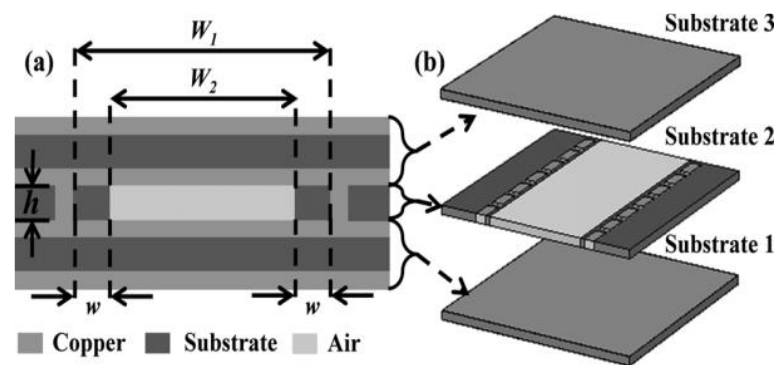

Figure 1. AFSIW (reproduced from [4])

\subsection{Wave propagation in SIW}

The analysis of wave propagation in dielectric-filled SIW is well known. Referring to Figure 2 for the case of constant $W$, the wave propagation is described as in a conventional rectangular waveguide, and thus, determining the cutoff frequency and subsequent dimensioning for the desired frequency bands is trivial. The cutoff frequency can be obtained using the classical waveguide equation [20] 


$$
f_{c m n}=\frac{c}{2 \sqrt{\varepsilon_{r}}} \sqrt{\left(\frac{m}{W}\right)^{2}-\left(\frac{n}{h}\right)^{2}}
$$

where $m$ and $n$ are the indices of $T E_{m n}$ mode, $c$ is the speed of light in free space, $\varepsilon_{r}$ is the substrate's relative permittivity, and $W$ is the width of the dielectric-filled SIW, given by

$$
W=\frac{\bar{W}}{\sqrt{\varepsilon_{r}}},
$$

where $\bar{W}$ is the width of a conventional rectangular waveguide.

A more complex case is in the transition, as depicted in Figure 2. Since not only is there a dielectric slab in the $E$-plane, the air-dielectric composition also varies along with the transition. The characteristics of wave propagation in a dielectric-loaded waveguide may be found in the study of Vartanian et al., where the characteristic equation is given as

$$
\tan \left(\frac{\sqrt{\varepsilon_{r}}\left(W_{1}-W_{2}\right) \pi f_{c}}{c}\right)=\sqrt{\varepsilon_{r}} \cot \left(\frac{W_{2} \pi f_{c}}{c}\right),
$$

where $W_{1}$ and $W_{2}$ are the total width and the width of the air-filled region, respectively, such that $W_{1}-W_{2}=2 w$ [15].

\subsection{Dimensions and parameters for SIW and AFSIW}

The SIW model consists of a dielectric-filled region and an air-filled region, with a transition in between. The dimensions and properties of the whole structure are designed in the Ka- and U-band frequencies. Referring to Figure 2, the total width in the air-filled region, i.e., the distance between the via-holes $W_{1}$ is calculated using (1), whereas the width of the dielectric-filled SIW $W$ is obtained using (2). From (2), $W=4.12$ $\mathrm{mm}$ for the Ka-band and $W=2.9 \mathrm{~mm}$ for the U-band. From (1), $W_{1}=7.02 \mathrm{~mm}$ for the Ka-band considering $w=0.508 \mathrm{~mm}$ and $W_{2}=6.0 \mathrm{~mm}$. For the U-band, considering the same value of $w$ and using $W_{2}=3.9 \mathrm{~mm}$, $W_{1}=4.9 \mathrm{~mm}$. These dimensions are calculated to obtain a constant $f_{c}=21.2 \mathrm{GHz}$ for the Ka-band and $f_{c}=30$ $\mathrm{GHz}$ for the U-band to avoid the high dispersive region occurring near the cut-off frequency [12]. All

\begin{tabular}{|c|c|c|c|c|}
\hline Band & \multicolumn{2}{|c|}{ Ka-band } & \multicolumn{2}{|c|}{ U-band } \\
\hline Parameters $(\mathrm{mm})$ & AFSIW & SIW & AFSIW & SIW \\
\hline$W_{1}$ & 7.02 & & 4.9 & \\
\hline$W_{2}$ & 6 & & 3.9 & \\
\hline$W$ & & 4.12 & & 2.9 \\
\hline$\varepsilon_{\mathrm{r}}$ & 2.94 & 2.94 & 2.94 & 2.94 \\
\hline$h$ & 0.508 & 0.508 & 0.254 & 0.254 \\
\hline$w$ & 0.508 & 0.508 & 0.508 & 0.508 \\
\hline
\end{tabular}
dimensions and properties of both dielectric-filled and air-filled regions are listed in Table 1.

Table 1. Structural properties of SIW

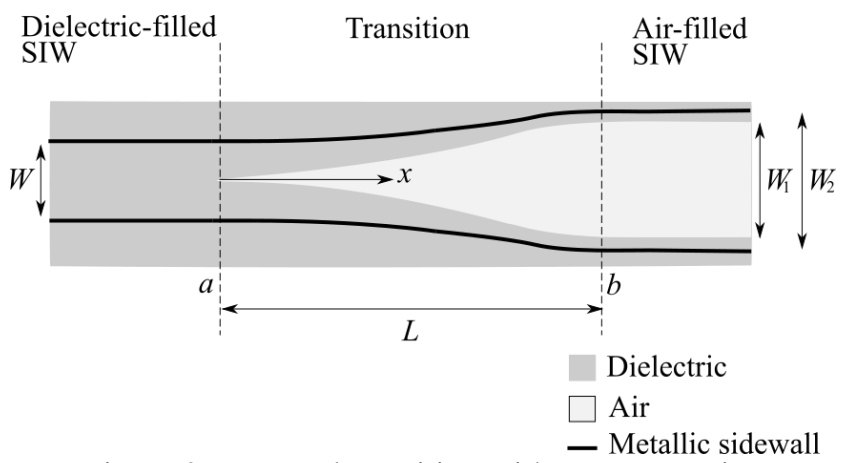

Figure 2. Tapered transition with a cross-section 


\subsection{Dielectric-filled to air-filled SIW transition}

To allow for an effective interconnect between the dielectric-filled region and the air-filled region of the SIW circuits, a transition must be carefully constructed [12-13]. As depicted in Figure 2, the width of the taper, $W_{2}$, increases along the transition length $L$; the widths at boundaries $a$ and $b$ of the transition are fixed to ensure the continuity of transition. Specifying $W_{2}(x)$ for $0 \leq x \leq L$ to ensure wave propagation along the transition length can be simplified by selecting $f_{c}$ at the start of transition and solving (3).

The influence of the taper profile is strongly implied in (3). While the solution of (3) for $W_{1}(x)$ and $W_{2}(x)$ ensures a wave propagation along the transition in the specified bandwidth and TE mode, optimality losses are not guaranteed. Comparing the approach with other closed-form functions of the taper profile, the profile of $W_{2}(x)$ with an assumed profile of $W_{1}(x)$ in (1) is not significantly better than the raised cosine profile for reduction of return and transmission losses along the AFSIW transition [21]. This observation agrees with the results obtained in [18-19], where the raised cosine taper profile is also desirable as a transition since the zero slopes at both ends ensure minimal reflections.

Another desirable consequence of (3) alludes to a specific width of the dielectric slab where the bandwidth, defined as the ratio of the cutoff frequency for the $\mathrm{TE}_{20}$ mode to that for the $\mathrm{TE}_{10}$ mode, can be extended [15]. Vartanian et al. suggested optimality of the dimension of the dielectric slab with respect to certain losses in the waveguide. In the context of varying width in a SIW transition, however, obtaining the optimal function $W_{2}(x)$ where losses are minimized is nontrivial since the search space for the specific geometry and length of the taper is infinite [11,15]. Consequently, a trade-off between total transition length and losses is usually made in practice [12]. The relation of transition length and taper design equation of transition losses has also been reported in [16-17].

In this study, we further develop the taper designs in [12] by defining the shape of the transition taper with the clamped cubic spline function in order to optimize the transition losses. By defining a spline, a fixed number of nodal points, or knots, defining the spline can serve as a finite number of unknowns to be optimized, as presented in Figure 3. Previous studies on the combination of the optimisation method with the spline method have been effective. For example, the optimisation of the spline-profile horn by using the quasi-Newton optimisation method is reported in [22]. Another study is smooth-walled spline-profile horn characteristic optimisation developed in [23]. More recently, an optimisation technique for microstrip antennas using cubic splines with particle swarm optimisation is presented in [24]. In this paper, an optimisation procedure for an AFSIW transition taper using clamped cubic splines with the multi-objective GA method is described.

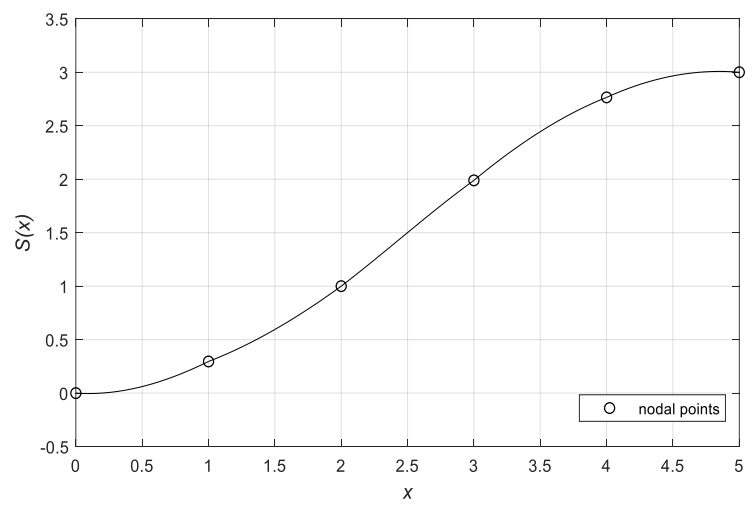

Figure 3. Taper profile with clamped cubic splines.

\subsection{Optimisation procedures}

Design optimisations using GAs in engineering electromagnetics are quite widespread in practice, for example, in antenna designs [25-26]. GA steps are described as follows [39]:

1. Initialize a random population of chromosomes.

2. Compute the fitness of each population member.

3. Rank individuals based on fitness.

4. Generate offspring by mating good individuals.

5. Mutate selected members of the offspring.

6. Stop if conditions have been met or continue back to step 2 . 
The electromagnetic optimisation problems generally involve several parameters, thus making the design process inefficient and difficult [27]. In the context of geometric optimisation of the transition between the air-filled and dielectric-filled regions, parameterising the shape of the transition with cubic splines is deemed to efficiently search the infinite possible shapes by using a relatively low number of parameters. Our approach is similar to those in [28-29]; the differences are in the use of clamped cubic splines and real-time data transfer between the GA module in Matlab and full-wave analysis in ANSYS HFSS. We further detail the optimisation procedures as follows:

The first step is specifying the design variables or phenotypes for the search space. By constructing the equation of the taper with the clamped cubic splines, as shown in Figure 3, the internal nodes provide four degrees of freedom $\left\{x_{1}, x_{2}, x_{3}, x_{4}\right\}$ as the phenotypes. These phenotypes comprise the "heights" of the knots. The initial height of each knot is set to closely match the raised cosine profile given by

$$
\frac{1}{2}(a+b)+\frac{1}{2}(b-a) \cos \left(\left[\frac{x}{L}-1\right] \pi\right)
$$

where the nominal values are matched to the operating bandwidth in either the Ka-band frequencies, i.e., 26 to $40 \mathrm{GHz}$, or U-band frequencies, i.e., 40 to $60 \mathrm{GHz}$. The upper and lower bounds of each point, i.e., $x \pm$ $0.16 \mathrm{~mm}$, are prescribed to avoid excessive distortions of the constructed taper shape.

The second step is setting up the GA procedure for multi-objective optimisation. The GA is used in this optimisation because of its efficiency and simplicity of implementation, especially in multimodal search spaces [30]. The fitness functions for the GA procedures introduced in this study aim to further minimize the return and transmission losses along with the transition, as achieved with the raised cosine geometry. In many applications, a return loss of $20 \mathrm{~dB}$ is commonly accepted for the target frequency band ranges of 26 to $40 \mathrm{GHz}$ for the Kaband and 40 to $60 \mathrm{GHz}$ for the $\mathrm{U}$ band. For fulfilling the first goal, a simple approach to define the fitness function involves taking the average of the reflection coefficient $S_{11}$ over the frequency band of interest. Hence, the first fitness function $F_{11}\left(x_{i}\right)$ reads

$$
F_{11}=\frac{1}{N} \sum_{i=1}^{N}\left|S_{11}\left(f_{i}\right)\right| \leq 20 d B
$$

where $f_{i}$ is the sampling frequency and $N$ is the number of samples. The fitness function $F_{21}\left(x_{i}\right)$ relates the criterion for the transmission loss where the average values of the transmission coefficient $S_{21}$ values over the frequency band of interest are set to be within

$$
F_{21}=\frac{1}{N} \sum_{i=1}^{N}\left|S_{21}\left(f_{i}\right)\right| \leq 0.1 d B .
$$

From observation, $F_{11}$ and $F_{21}$ are most of the time mutually conflicted. For multiple conflicting objectives, multi-objective optimisation resorts to several trade-offs optimal solutions [31]. Different multiobjective GAs are available, such as niched Pareto GA, non-dominated sorting GA (NSGA), and strength Pareto evolutionary algorithm, with variations in selection methods and diversity control techniques [32-34]. Our optimisation employs a variant of NSGA, NSGA-II, owing to its fast non-dominated sorting procedure and its simple yet efficient constraint-handling method [35]. The strategy for the combination of GA and full-wave simulation is illustrated in Figure 4.

The single-objective GA and multi-objective GA generate different individuals that describe the transition profile with splines, which directly influence the return and transmission losses from the full-wave analysis. In practice, the resulting multi-objective GA optimisation produces a set of Pareto-optimal solutions and the final solution is largely a matter of arbitrary selection [36]. In this optimisation procedure, the solution with minimal losses is selected. To better understand the effect of transition length and the effectiveness of optimisation for shorter lengths, numerical studies are performed for selected lengths: 5, 10, 15, and $20 \mathrm{~mm}$. A transition length of $20 \mathrm{~mm}$ is commonly accepted to provide acceptable losses in practice [12].

Hence, it is of major interest to further minimize losses at a shorter transition length to allow the possibility of a more compact SIW design. The procedure for multi-objective optimisation with GA described above is implemented in Matlab, while the full-wave analysis is computed with ANSYS HFSS. The interface code for real-time data transfer between both packages is written in Visual Basic. 


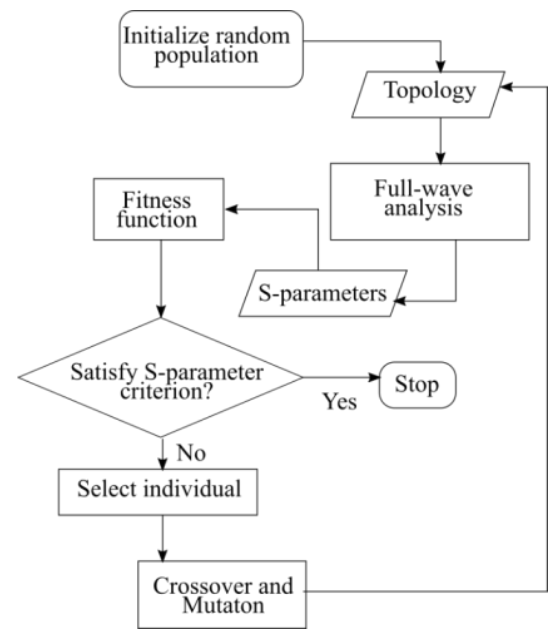

Figure 4. Flowchart of GA optimisation with full-wave analysis.

\section{RESULTS AND DISCUSSION}

The results of the optimisation for the operating frequencies in the Ka (26 to $40 \mathrm{GHz}$ ) and $\mathrm{U}$ (40 to 60 $\mathrm{GHz}$ ) bands and at the previously mentioned transition lengths are presented. Single objective (SO) GA is also performed for comparison with multi-objective (MO) GA. Tables 2 and 3 show the final phenotypes with MOGA optimisation for the two frequency bands, while Figs. 5 and 6 show the resulting S-parameters from the optimized phenotypes for a $5 \mathrm{~mm}$ transition length at the Ka-band frequency. Moreover, the results of optimization have been compared with the results of arbitrarily to assure that the designed waveguide has been optimized as depicted in Figs. 5 and 6. Clearly, the new transition profile constructed from the optimized phenotypes shows an improvement in the losses.

The MO transition taper yields the best performance with overall lower return loss $S_{11}$ between 17.38 and $48.68 \mathrm{~dB}$ within a frequency range of 26 to $40 \mathrm{GHz}$. Based on a comparison of the maximum $S_{11}$ results, the performance of the MO taper design is better compared to taper design in [12]. The significant improvement in the region around $36 \mathrm{GHz}$ compared to the raised cosine profile indicates the effectiveness of MO optimisation. Some improvements can also be observed in the transmission loss $S_{21}$.

Table 2. Optimized phenotypes obtained using MO optimisation in Ka-band

\begin{tabular}{cccccc}
\hline & \multicolumn{4}{c}{ Length, mm } & \multicolumn{4}{c}{ Phenotypes, mm } \\
\hline & & $x_{1}$ & $x_{2}$ & $x_{3}$ & $x_{4}$ \\
Initial & & 0.2865 & 1.0365 & 1.9635 & 2.7135 \\
Optimized & $\mathbf{5}$ & 0.2958 & 1.0 & 1.9892 & 2.7651 \\
& $\mathbf{1 0}$ & 0.2942 & 1.0027 & 1.8070 & 2.7506 \\
\hline
\end{tabular}

Table 3. Optimized phenotypes obtained using MO optimisation in U band

\begin{tabular}{cccccc}
\hline & Length, mm & \multicolumn{4}{c}{ Phenotypes, mm } \\
\hline & & $x_{1}$ & $x_{2}$ & $x_{3}$ & $x_{4}$ \\
\hline Initial & & 0.1862 & 0.6737 & 1.2763 & 1.7638 \\
Optimized & $\mathbf{5}$ & 0.1786 & 0.6199 & 1.1743 & 1.622 \\
& $\mathbf{1 0}$ & 0.1741 & 0.62 & 1.1876 & 1.6239 \\
\hline
\end{tabular}

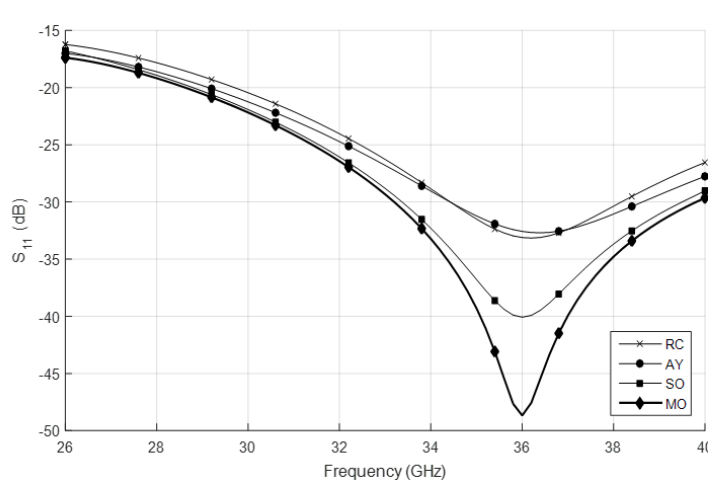

Figure 5. Comparison of $\left|\mathrm{S}_{11}\right|$ at $\mathrm{L}=5 \mathrm{~mm}$ in the Ka-band frequency at $33 \mathrm{GHz}$ : RC= Raised Cosine, GA = Single-objective GA, MO-GA = Multi-objective GA, AY= Arbitrary method. 


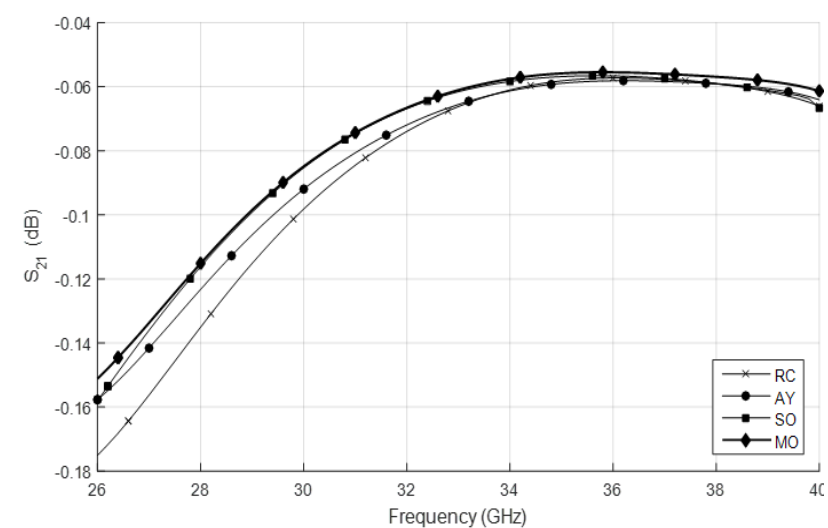

Figure 6. Comparison of $\left|\mathrm{S}_{21}\right|$ at $\mathrm{L}=5 \mathrm{~mm}$ in the Ka-band frequency at $33 \mathrm{GHz}$ : RC= Raised Cosine, GA = Single-objective GA, MO-GA = Multi-objective GA, AY= Arbitrary method.

For the $10 \mathrm{~mm}$ transition length, the resulting S-parameters are depicted in Figs. 7 and 8. It is apparent that although both optimisation procedures improve the losses from the raised cosine taper profile, there is no significant advantage of multi-objective GA over the single-objective one.

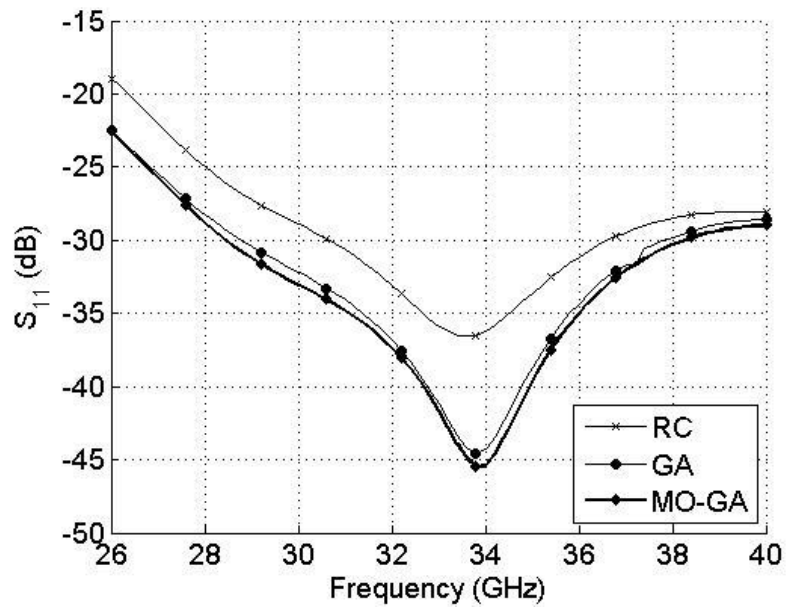

Figure 7. Comparison of $\left|\mathrm{S}_{11}\right|$ at $\mathrm{L}=10 \mathrm{~mm}$ in the Ka-band frequency at $33 \mathrm{GHz}$ : $\mathrm{RC}=$ Raised Cosine, GA = Single-objective GA, MO-GA = Multi-objective GA

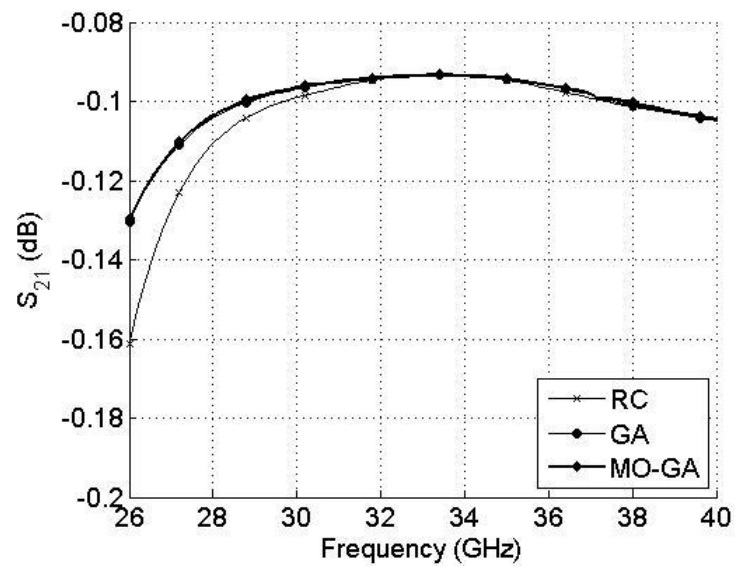

Figure 8. Comparison of $\left|\mathrm{S}_{21}\right|$ at $\mathrm{L}=10 \mathrm{~mm}$ in the Ka-band frequency at $33 \mathrm{GHz}$ : $\mathrm{RC}=$ Raised Cosine, GA = Single-objective GA, MO-GA = Multi-objective GA 
A similar significant improvement of MO-GA optimisation on the transition taper is observed for the Uband frequencies where the $5 \mathrm{~mm}$ transition length is observed to gain the highest improvement of the reflection loss, as depicted in Figs. 9 and 10. The reflection coefficient $S_{11}$ values of 12.51 to $34.89 \mathrm{~dB}$ from $40 \mathrm{to} 60 \mathrm{GHz}$ and the transmission coefficient $S_{21}$ values of 0.165 to $0.160 \mathrm{~dB}$ from 50 to $60 \mathrm{GHz}$ are obtained.

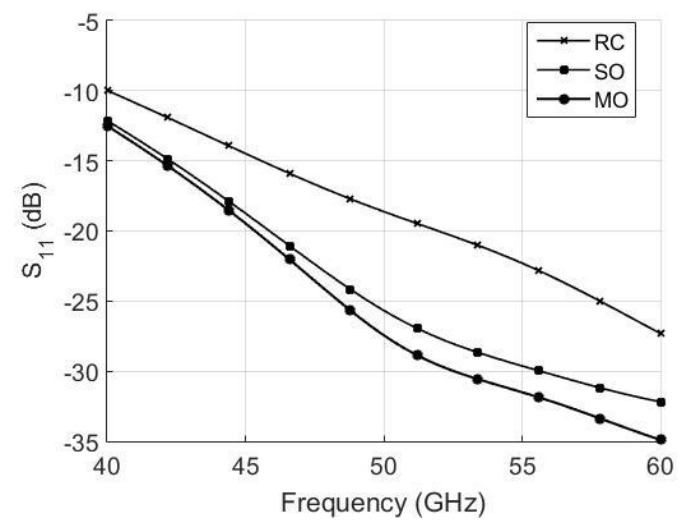

Figure 9. Comparison of $\left|\mathrm{S}_{11}\right|$ at $\mathrm{L}=5 \mathrm{~mm}$ in the U-band frequency at $50 \mathrm{GHz}$ : RC $=$ Raised Cosine, $\mathrm{SO}=$ Single-objective GA, MO = Multi-objective GA

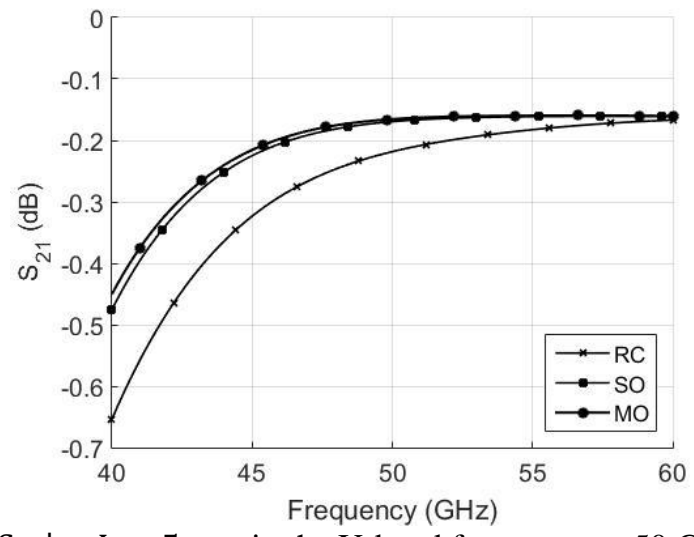

Figure 10. Comparison of $\left|\mathrm{S}_{21}\right|$ at $\mathrm{L}=5 \mathrm{~mm}$ in the U-band frequency at $50 \mathrm{GHz}$ : RC $=$ Raised Cosine, $\mathrm{SO}=$ Single-objective GA, MO = Multi-objective GA

The resulting S-parameters from the optimisation of the taper with a $10 \mathrm{~mm}$ transition length in the Uband frequencies are depicted in Figs. 11 and 12. In general, MO optimisation can further reduce the return loss for the bandwidth between 52 and $60 \mathrm{GHz}$. More significant improvement occurs in the transmission loss, where both optimisation procedures can improve the raised cosine profile.

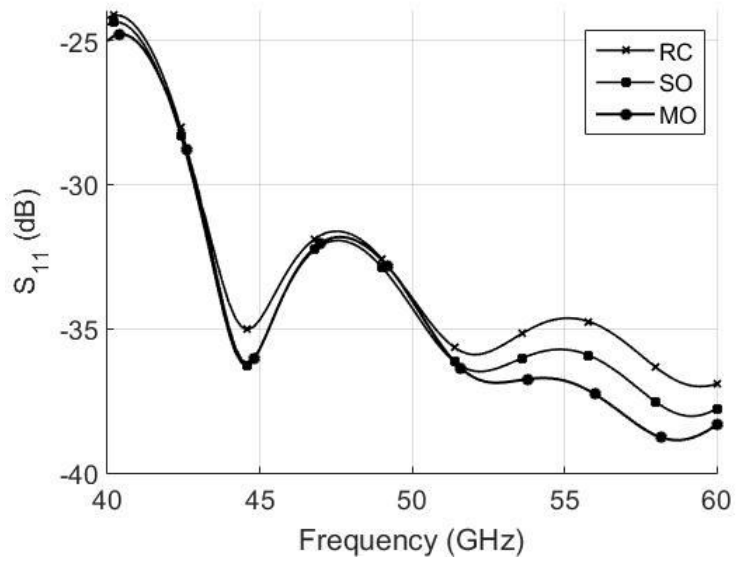

Figure 11. Comparison of $\left|\mathrm{S}_{11}\right|$ at $\mathrm{L}=10 \mathrm{~mm}$ in the U-band frequency at $50 \mathrm{GHz}$ : RC= Raised Cosine, $\mathrm{SO}=$

Single-objective GA, MO-GA = Multi-objective GA 


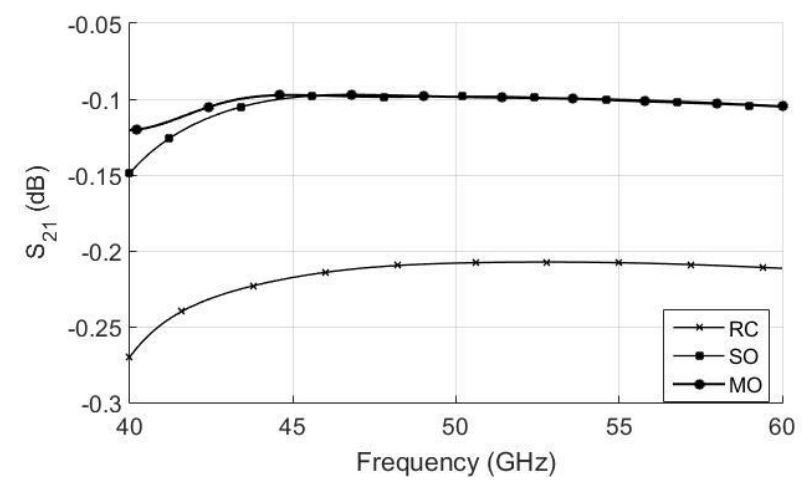

Figure 12. Comparison of $\left|\mathrm{S}_{21}\right|$ at $\mathrm{L}=10 \mathrm{~mm}$ in the $\mathrm{U}$-band frequency at $50 \mathrm{GHz}$ : $\mathrm{RC}=$ Raised Cosine, $\mathrm{SO}=$ Single-objective GA, MO = Multi-objective GA

As listed in Table 4, the overall return and insertion loss improvements, computed using the maximum values of losses within the bandwidth, for the Ka-band frequencies are apparent. A significant maximum return loss can be obtained. At different transition lengths, particularly for shorter transitions such as 5 and $10 \mathrm{~mm}$, such an improvement may benefit in allowing a more compact SIW design. Nevertheless, for the $10 \mathrm{~mm}$ transition length, insertion loss and bandwidth improvement are not as significant. Similarly, Table 5 summarises the return and insertion loss improvements for the U-band frequencies. Of note is the significant reduction in insertion loss obtained with the MO-GA procedure for all selected transition lengths.

Table 4. Percentage of the return and insertion loss improvements of the optimised taper to the RC taper

\begin{tabular}{|c|c|c|c|c|}
\hline & \multicolumn{4}{|c|}{ Length, mm } \\
\hline & 5 & 10 & 15 & 20 \\
\hline & \multicolumn{4}{|c|}{ Return loss } \\
\hline SO & $21.2 \%$ & $21.7 \%$ & $35.8 \%$ & $5.9 \%$ \\
\hline \multirow[t]{2}{*}{ MO } & $45 \%$ & $24.1 \%$ & $46.8 \%$ & $10.5 \%$ \\
\hline & \multicolumn{4}{|c|}{ Insertion loss } \\
\hline SO & $1.2 \%$ & $0.3 \%$ & $0.4 \%$ & $0.4 \%$ \\
\hline MO & $3.1 \%$ & $1.1 \%$ & $0.5 \%$ & $0.5 \%$ \\
\hline
\end{tabular}

Table 5. Percentage of the return and insertion loss improvements of the optimised taper to the RC taper (U-band)

\begin{tabular}{|c|c|c|c|c|}
\hline & \multicolumn{4}{|c|}{ Length, mm } \\
\hline & 5 & 10 & 15 & 20 \\
\hline & \multicolumn{4}{|c|}{ Return loss } \\
\hline SO & $3.4 \%$ & $0.5 \%$ & $0 . \%$ & $0.1 \%$ \\
\hline \multirow[t]{2}{*}{ MO } & $4.0 \%$ & $0.6 \%$ & $0.4 \%$ & $0.1 \%$ \\
\hline & \multicolumn{4}{|c|}{ Insertion loss } \\
\hline SO & $17.8 \%$ & $2.8 \%$ & $17.9 \%$ & $22.6 \%$ \\
\hline MO & $27.8 \%$ & $5.0 \%$ & $48.3 \%$ & $47.4 \%$ \\
\hline
\end{tabular}

\subsection{Wideband characteristics in SIW transition}

In many applications, it is of major interest to acquire a SIW design where the losses are as low as possible for broad bandwidth. Although no fitness function is constructed to account for the bandwidth optimisation, the changes with respect to the bandwidth operation resulting from the current optimisation scheme are herewith presented for comparison. From the definition of bandwidth:

$$
B W=f_{2}-f_{1}
$$

where $f_{1}, f_{2}$ are the lower and upper-frequency limits, respectively, the wideband characteristic is deemed achieved if the bandwidth below $20 \mathrm{~dB}$ is at least $11.2 \mathrm{GHz}$. Defining in terms of percentage fractional bandwidth as in [14] 


$$
\% F B W=\frac{f_{2}-f_{1}}{f_{o}} \times 100 \%
$$

with $f_{o}$ as a design center frequency, the wideband performance of the optimized designs can thus be characterised.

For the $5 \mathrm{~mm}$ transition in the Ka-band, the optimised transition with MO-GA shows an almost $34 \%$ improvement in wideband characteristics relative to the raised-cosine transition. For the $10 \mathrm{~mm}$ transition, the improvement is even larger, i.e., about 42\%. Observing the resulting S-parameters in the U band, similar improvements have been acquired. For the $5 \mathrm{~mm}$ transition, an improvement of almost $28 \%$ is observed, whereas the $10 \mathrm{~mm}$ transition shows about $40 \%$ improvement. Although unintended, such improvement encourages the addition of another fitness function to the current multi-objective optimisation scheme, which can be easily implemented.

\section{CONCLUSION}

From the computational analysis above, we can conclude that the above procedures are effective in finding an optimal transition taper, where the new taper transition geometry constructed from the clamped cubic spline is shown to further minimize the return and transmission losses. The optimisation strategy also shows the efficacy of real-time coupling between MATLAB for its optimisation procedures and ANSYS HFSS for its fullwave electromagnetic simulations. The successful coupling provides more options for further optimisations with respect to the variable data pertaining to the electromagnetic wave propagation in an SIW taper and other microwave devices. Furthermore, the findings of the present study may contribute to the development of more compact design of coplanar circuits for any frequency band with excellent performance.

\section{REFERENCES}

[1] D. Deslandes and K. Wu, "Accurate modeling, wave mechanisms, and design considerations of a substrate integrated waveguide”, IEEE Trans. Microw. Theory Tech., vol. 54, no.6, pp. 2516-2526, 2006.

[2] D. Deslandes and K. Wu, "Integrated microstrip and rectangular waveguide in the planar form", IEEE Microw. Compon. Lett., vol. 11, no.2, pp. 68-70, 2001.

[3] Y. Cassivi and L. Perregrini et al, "Dispersion characteristic of substrate integrated rectangular waveguide", IEEE Microw. Wireless Compon. Lett., vol.12, no.9, pp. 333-334, 2002.

[4] M.N.M. Kehn, "Modal analysis of substrate integrated waveguides with rectangular via-holes using cavity and multilayer Green's functions", IEEE Trans. Microw. Theory Tech., vol. 62, no.10, pp. 2214-2231, 2014.

[5] W. Che and K. Deng et al, "Analytical equivalence between substrate-integrated waveguide and rectangular waveguide', IET Microw. Antennas Propag., vol. 2, no.1, pp. 35-41,2008.

[6] M. Bozzi, L. Perregrini and P. Arcioni, "Current and future research trends in substrate integrated waveguide technology", Radioengineering, vol.18, no.2, pp. 201-209, 2009.

[7] M. Bozzi, L. Perregrini and K. Wu, "Modeling of conductor, dielectric, and radiation losses in substrate integrated waveguide by the boundary integral-resonant mode expansion method", IEEE Trans. Microw. Theory Tech., vol. 56, no.12, pp. 3153-3161, 2008.

[8] Q. Lai, C. Fumeaux, W. Hong and R. Vahldieck, "Characterization of the propagation properties of the half-mode substrate integrated waveguide", IEEE Trans. Microw. Theory Tech., vol. 57, no.8, pp. 1996-2004, 2009.

[9] N. Ranjkesh and M. Shahabadi, "Reduction of dielectric losses in substrate integrated waveguide", Electronics Letters, vol. 42, no.21, pp. 1230-1231, 2006.

[10] L. Jin, R. M. A. Lee and I. Robertson, "Analysis and design of a novel low-loss hollow substrate integrated waveguide”, IEEE Trans. Microw. Theory Tech., vol. 62, no.8, pp. 1616-1624, 2014.

[11] A. Belenguer and A.L. Borja et al, "High-performance coplanar waveguide to empty substrate integrated coaxial line transition”, Trans. Microw. Theory Tech., vol. 63, no.12, pp. 4027-4034, 2015.

[12] F. Parment and A. Ghiotto et al, "Air-filled substrate integrated waveguide for low-loss and high power-handling millimetre-wave substrate integrated circuits", IEEE Trans. Microw. Theory Tech., vol. 63, no.4, pp. 1228-1238, 2015.

[13] N. Ghassemi and I. Boudreau et al, "Millimetre-wave broadband transition of substrate integrated waveguide on high-tolow dielectric constant substrates”, IEEE Trans. Compon., Packag. Manuf. Technol., vol.3, no.10, pp. 1764-1770, 2013.

[14] J.D. Kraus and D.A. Fleisch, Electromagnetics with applications. McGraw-Hill, 1999.

[15] P.H. Vartanian, W.P. Ayres and A.L. Helgesson, "Propagation in dielectric slab loaded rectangular waveguide", IRE Trans. Microw. Theory Tech., vol.6, no. 2, pp. 215-222, 1958. 
[16] R.P. Hecken and A. Anuff, "On the optimum design of tapered waveguide transitions", IEEE Trans. Microw. Theory Tech., vol.21, no.6, pp. 374-380, 1973.

[17] R.P. Hecken and A. Anuff, "Near-optimum matching section without discontinuities", IEEE Trans. Microw. Theory Tech., vol. 20, no.11, pp. 734-739. 1972.

[18] D.S. Nagarkoti and R. Sharma et al, "Analysis of nonlinear cylindrical waveguide taper using modal matching technique”, Int. J. Microw. Appli., vol. 1, no.1, pp. 5-12, 1985.

[19] H.G. Unger, “Circular waveguide taper of improved design”, Bell System Tech. J., vol.37, no.4, pp. 877-898, 1958.

[20] Y. Cassivi and L. Perregrini et al, "Dispersion characteristics of substrate integrated rectangular waveguide", IEEE Microw. Compon. Lett., vol.12, no.9, pp. 333-335, 2002.

[21] H. Mansor, and R. Abdul-Rahman, "Optimal transition in air-filled substrate integrated waveguide", Proc. IEEE Stud. Conf. Res. Dev., Kuala Lumpur, Malaysia, pp. 172-176, 2016.

[22] C. Granet, G.L. James, "A smooth-walled spline-profile horn as an alternative to the corrugated horn for wide band millimetre-wave applications", IEEE Trans. Antennas Propag., vol.52, no.3, pp. 848-854, 2004.

[23] N. Popenko and R. Chernobrovkin et al, "Spline-profile smooth-walled horn as a feed of the multi-beam millimetre focal plane array", Proc. 15th IEEE Mediterranean Electrotech. Conf., Valletta, Malta,pp. 1278-1282, 2010.

[24] M. Moniruzzaman and M.R. Islam et al, "Cubic spline based particle swarm optimisation for microstrip antennas", Australian J. Basic Appl. Sci., vol.5, no.12, pp. 804-807, 2011.

[25] M.T. Asghar and M.F. Shafique et al, "Design and optimisation of an UWB antenna with $5.8 \mathrm{GHz}$ band suppression using genetic algorithm”, J. Basic. Appl. Sci. Res., vol.3, no.7, pp. 701-707, 2013.

[26] D. Zhou and R.A. Abd-Alhameed et al, "New antenna designs for wideband harmonic-suppression using adaptive surface meshing and genetic algorithms", IET Microw. Antennas Propag., pp. 1054-1061, 2011.

[27] Y. Rahmat-Samii and E. Michielssen, Electromagnetic optimisation by genetic algorithms. John Wiley \& Sons, 1999.

[28] S. Shuhui and W. Bingzhong, "Parameter optimisation based on GA and HFSS", J. Electron. Sci. Tech. China, vol.3, pp. 45-47, 2005.

[29] H. Choo and H. Ling, "Design of broadband and dual-band microstrip antennas on high dielectric substrate using the genetic algorithm”, IEEE Proc. Microw. Antennas Propagat., vol. 150, no.3, pp. 137-142, 2003.

[30] S. Bandyopadhyay and S. Saha, Unsupervised classification. Springer, 2013.

[31] K. Deb, Multi-objective optimisation using evolutionary algorithms. John Wiley \& Sons, 1999.

[32] J. Horn, N. Nafploitis and D.E. Goldberg, "A niched Pareto genetic algorithm for multi-objective optimization”, Proc. the First IEEE Conf. on Evol. Comp., Orlando, FL, USA, pp. 82-87, 1994.

[33] J. Knowles and D. Corne, "The Pareto archived evolution strategy: a new baseline algorithm for multi-objective optimization”, Proc. 1999 Congress on Evol. Comp., Washington, DC, USA, pp. 98-105, 1999.

[34] N. Srinivas and K. Deb, "Multi-objective function optimisation using non-dominated sorting genetic algorithms", Evol. Comput., vol.2, no.3, pp. 221-248, 1995.

[35] K. Deb and A. Pratap et al, "A fast and elitist multi-objective genetic algorithm: NSGA-II", IEEE Trans. Evol. Comput., vol. 6, no.2, pp. 182-197, 2002.

[36] S. Rao, Engineering optimisation theory and practice. John Wiley \& Sons, 2008. 